\title{
TREINAMENTO AERÓBIO E RESISTIDO, QUALIDADE DE VIDA E CAPACIDADE FUNCIONAL DE HIPERTENSAS
}

\author{
AEROBIC AND RESISTANCE TRAINING, QUALITY OF LIFE, FUNCTIONAL CAPACITY \\ IN HYPERTENSIVE WOMEN
}

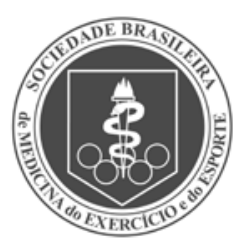

Artigo Original

\author{
ENTRENAMIENTO AERÓBICO Y RESISTIDO, CALIDAD DE VIDA Y CAPACIDAD \\ FUNCIONAL EN HIPERTENSAS
}

\begin{abstract}
Thaís Inacio Rolim Póvoa
(Educadora Física) ${ }^{1}$

Paulo César Brandão Veiga Jardim

(Médico Cardiologista)2.

Ana Luiza Lima Sousa

$(\text { Enfermeira })^{2}$

Thiago de Souza Veiga Jardim

(Médico Cardiologista) $^{2}$

Weimar Kunz Sebba Barroso

de Souza (Médico Cardiologista)²

Luciana Sanches Veiga Jardim

(Médica Endocrinologista) ${ }^{2}$

1. Escola Superior de Educação Física e Fisioterapia de Goiás (ESEFFEGO) da Universidade Estadual de Goiás, Goiânia, GO, Brasil.

2. Liga de Hipertensão Arterial da Faculdade de Medicina da Universidade Federal de Goiás. Goiânia, GO, Brasil.
\end{abstract}

\section{Correspondência:}

Rua Dona Stela, n 151, Bl 1, Apto 404. Residencial Portal das Veredas. Setor Negrão de Lima, Goiânia, GO, Brasil. 74650-100. thaisrpovoa@gmail.com

\section{RESUMO}

Introdução: Pacientes hipertensos podem apresentar comprometimento da qualidade de vida (QV) e da qualidade de vida relacionada à saúde (QVRS), tanto pela hipertensão arterial (HA), quanto pelos eventuais efeitos adversos do tratamento. Exercícios físicos, aeróbios e resistidos, melhoram a performance cardiorrespiratória e neuromuscular, mas há poucas evidências sobre seus efeitos na QV, QVRS e capacidade funcional (CF) em mulheres hipertensas. Objetivo: Avaliar e comparar os efeitos dos treinamentos aeróbio e resistido sobre a QV, QVRS e a capacidade funcional em hipertensas. Métodos: Ensaio clínico randomizado, cego, com total de 18 sessões de exercícios. Foram incluídas mulheres hipertensas sob tratamento medicamentoso, não participantes de programas de exercícios, com 50 anos de idade ou mais, que não apresentaram arritmias e/ou alterações isquêmicas em teste ergométrico (protocolo de Bruce). A amostra foi randomizada como segue: grupo aeróbio (GA) ( $n=21)$ e grupo resistido (GR) $(n=20)$. Intensidade GA: leve a moderada (Escala de Borg adaptada); GR: até 50-65\% de 1 RM. Duas séries com 12 a 15 repetições. Antes e após a intervenção foram aplicados os questionários WHOQOL-bref (QV) e SF-36 (QVRS) e realizado o teste de caminhada de 6 minutos (TC6') para avaliar a CF. Estatística: testes Shapiro-Wilk, $t$ de Student, Fisher, $U$ de Mann-Whitney e Wilcoxon. Nível de significância: $p<0,05$. Resultados: Os grupos eram inicialmente homogêneos nos aspectos clínicos, antropométricos, funcionais e sociodemográficos ( $p \geq 0,05)$. Após a intervenção houve melhora significativa em todos os domínios do WHOQOL-bref no GA e no GR houve melhora no domínio aspectos físicos. No SF-36, constatou-se melhora significativa em sete de oito domínios tanto no GA quanto no GR. Capacidade funcional: verificou-se melhora nos dois grupos $(p \leq 0,001)$. Conclusão: Os dois tipos de treinamento melhoraram a QV e a CF e, dependendo dos objetivos estabelecidos, ambos podem ser eficazes.

Palavras-chave: hipertensão arterial, exercício aeróbico, treinamento de resistência, qualidade de vida.

\section{ABSTRACT}

Introduction: Hypertensive patients may have compromised quality of life (QoL) and health-related quality of life (HRQoL) because of arterial hypertension (AH) and for possible adverse effects of treatment. Physical, aerobic and resistance exercises, improve cardiorespiratory and neuromuscular performance, but there is little evidence about their effects on QoL, HRQoL and functional capacity (FC) in hypertensive women. Objective: To evaluate and compare the effects of aerobic and resistance training on QoL, HRQoL and functional capacity in hypertensive. Methods: A randomized, blinded clinical trial, with a total of 18 exercise sessions. Hypertensive women receiving drug treatment, non participant in exercise programs, with 50 years of age or more, which did not show arrhythmias and/or ischemic changes on ergometric testing (Bruce protocol) were included. The sample was randomized as follows: aerobic group (AG) $(n=21)$ and resistance group $(R G)(n=20)$. AG intensity: mild to moderate (Borg Scale adapted); $R G$ : up to 50-65\% of 1 MR. Two series of 12 to 15 repetitions. Before and after the intervention the WHOQOL-BREF and SF-36 (HEQOL) questionnaires were applied and the 6-minute walk test (6 ' MWT) was performed to evaluate the FC. Statistics: Shapiro-Wilk, Student t, Fisher, Mann-Whitney $\cup$ and Wilcoxon tests. Level of significance: $p<0.05$. Results: The groups were initially homogeneous in clinical, anthropometric, sociodemographic and functional aspects $(p \geq 0.05)$. After the intervention, there was significant improvement in all domains of WHOQOL-bref in $A G$, and in $R G$ an improvement of the physical domain was found. In the SF-36, there was significant improvement in seven of eight domains in both AG and RG. Functional capacity: improvement was found in both groups $(p \leq 0.001)$. Conclusion: The two types of training improved $Q \mathrm{~L}$ and functional capacity, and depending on the objectives established, both can be effective.

Keywords: hypertension, aerobic exercise, resistance exercise, quality of life.

\section{RESUMEN}

Introducción: Los pacientes hipertensos pueden presentar limitaciones en la calidad de vida (QV) y en la calidad de vida relacionada a la salud (QVRS), tanto por la hipertensión arterial (HA), como por los eventuales efectos adversos del tratamiento. Los ejercicios físicos, aeróbicos y resistidos mejoran el desempeño cardiorrespiratorio y neuromuscular, pero hay pocas evidencias sobre sus efectos en la QV, QVRS y capacidad funcional (CF) en mujeres hipertensas. Objetivo: Evaluar y comparar los efectos de los entrenamientos aeróbico y resistido sobre la QV, QVRS y la capacidad funcional en hipertensas. Métodos: Ensayo clínico aleatorizado, ciego, con total de 18 sesiones de 
ejercicios. Fueron incluidas mujeres hipertensas bajo tratamiento medicamentoso, no participantes en programas de ejercicios, con 50 años de edad o más, que no presentaron arritmias y/o alteraciones isquémicas en test ergométrico (protocolo de Bruce). La muestra fue aleatorizada como sigue: grupo aeróbico (GA) ( $n=21)$ y grupo resistido (GR) (n = 20). Intensidad GA: Iiviana a moderada (Escala de Borg adaptada); GR: hasta 50-65\% de 1 RM. Dos series con 12 a 15 repeticiones. Antes y después de la intervención fueron aplicados los cuestionarios WHOQOL-bref (QV) y SF-36 (QVRS) y realizado el test de caminata de 6 minutos (TC6') para evaluar la CF. Estadística: tests Shapiro-Wilk, t de Student, Fisher, U de Mann-Whitney y Wilcoxon. Nivel de significancia: $p<0,05$. Resultados: Los grupos eran inicialmente homogéneos en los aspectos clínicos, antropométricos, funcionales y sociodemográficos ( $p \geq 0,05)$. Después de la intervención hubo mejora significativa en todos los dominios de WHOQOL-bref en el GA y en el GR hubo mejora en el dominio aspectos físicos. En el SF-36, se constató mejora significativa en siete de ocho dominios tanto en el GA como en el GR. Capacidad funcional: se verificó mejora en los dos grupos $(p \leq 0,001)$. Conclusión: Los dos tipos de entrenamiento mejoraron la QV y la CFy, dependiendo de los objetivos establecidos, ambos pueden ser eficaces.

Palabras clave: hipertensión arterial, ejercicio aeróbico, entrenamiento de resistencia, calidad de vida.

Artigo recebido em 10/03/2013, aprovado em 23/01/2014

\section{INTRODUÇÃO}

A hipertensão arterial (HA) é fator de risco independente para doenças cardiovasculares (DCV) e constitui um problema de saúde pública mundial ${ }^{1-2}$. Pareceres institucionais $s^{3-5}$, dentre eles um específico para prevenção de DCV em mulheres ${ }^{3}$, estimulam a prática de exercícios físicos aeróbios e resistidos como terapia não medicamentosa para a prevenção ou tratamento da HA e de DCV por proporcionarem ganhos cardiocirculatórios e neuromotores.

Há evidências significativas de que os exercícios aeróbios promovem redução na pressão arterial (PA) subaguda e crônica, especialmente em hipertensos ${ }^{6-8}$. Já nos exercícios resistidos têm-se visto resultados ainda controversos (ora redução ora manutenção ou elevação da PA imediatamente após cada sessão). Estudos ${ }^{9-11}$ verificaram que tende a ocorrer hipotensão pós-exercício resistido em hipertensos, em um período após cerca de 10 minutos da sessão ou até 12 horas após, o que assinala que este tipo de exercício pode ser seguro ao paciente hipertenso ${ }^{12}$. É também importante por melhorar valências da aptidão física como a força, potência, hipertrofia e resistência muscular localizada, que somadas à função cardiorrespiratória incidem diretamente na capacidade funcional. Estes dados têm colocado o exercício resistido no rol de estratégias complementares ao hipertenso ${ }^{4-5}$.

Em relação aos efeitos dos exercícios aeróbios e/ou resistidos sobre a qualidade de vida (QV) e qualidade de vida relacionada à saúde (QVRS), há escassez de estudos. Há, no entanto, alguns indícios de que a QV tem forte relação com a capacidade funcional, ou seja, sujeitos com menos limitações e mais autonomia funcional tendem a ter mais satisfação de vida, melhor capacidade para enfrentar o estresse, a tensão, mais saúde, mais alegria, autoestima e, consequentemente, melhor $\mathrm{QV}^{13}$.

Embora a HA seja comumente considerada uma doença assintomática, os hipertensos podem apresentar pior QV e QVRS, pela própria doença, por comorbidades ou por eventuais efeitos adversos do tratamento medicamentoso. Estudos ${ }^{14-15}$ mostraram associação da HA com dores de cabeça, tonturas e cansaço, sendo que hipertensos, em questionários de qualidade de vida, parecem ter piores escores de estado geral de saúde em comparação a normotensos e a pessoas sem doenças crônicas não transmissíveis (DCNT's). A avaliação da QV em hipertensos pode ser uma ferramenta importante para estimar a efetividade de intervenções ou de prováveis mudanças na percepção dos pacientes com relação aos efeitos das doenças.

Este estudo objetivou avaliar e comparar os efeitos dos treinamentos aeróbio (caminhada) e resistido, realizados em um parque público de uma capital brasileira, sobre a QV, QVRS e capacidade funcional em hipertensas, com protocolos de treinamento com metodologias viáveis e mais acessíveis à população.

\section{MATERIAL E MÉTODOS}

O estudo foi realizado após aprovação de seu projeto pelo Comitê de Ética em Pesquisa Humana e Animal do Hospital das Clínicas da UFG (Protocolo: 077/2008). O termo de Consentimento Livre e Esclarecido (TCLE) foi aplicado anteriormente à realização dos procedimentos de coleta de dados e da intervenção.

Trata-se de um ensaio clínico randomizado, realizado na Liga de Hipertensão Arterial (LHA) da Universidade Federal de Goiás (UFG) e em um parque público da cidade de Goiânia, GO, Brasil.

Feito cálculo do número amostral e o número de 20 pacientes para cada grupo foi suficiente para detectar uma diferença de 35 pontos (35\%) nos escores do SF-36, considerando os valores descritos por Ciconelli ${ }^{16}$ e de 12 pontos (12\%) nos escores do WHOQOL-bref, descritos por Fleck ${ }^{17}$, com um nível de significância de $5 \%$ e poder de $80 \%$.

Critérios de inclusão: hipertensão arterial, sexo feminino, idade $\geq$ 50 anos, matrícula e tratamento multidisciplinar na LHA/UFG em um período mínimo de seis meses, não participação em programas de exercícios (mínimo de três meses), pressão arterial controlada e estável (sem mudança na medicação em um período prévio mínimo de seis meses) e teste ergométrico sem arritmias e/ou alterações isquêmicas. Critérios de exclusão: Infarto do miocárdio recente (menos de seis meses); acidente vascular cerebral recente (menos de seis meses) ou incapacitante; insuficiência cardíaca congestiva; insuficiência renal crônica; diabetes descompensado, doenças neuromusculares, musculoesqueléticas ou articulares não tratadas e em estágio grave e qualquer outra doença ou limitação que pudesse comprometer a realização dos protocolos de exercício físico. Tais parâmetros foram verificados nos prontuários do serviço.

Após a verificação dos critérios de inclusão e exclusão, foi feita randomização das pacientes pré-selecionadas $(n=55)$ para dois grupos: aeróbio (GA), em que foram alocadas 28 pacientes e grupo resistido $(G R)$, alocadas 27. Todas assinaram o Termo de Consentimento Livre e Esclarecido (TCLE) e em seguida foram submetidas à avaliação clínica por médico cardiologista, tendo sido retiradas do estudo oito pacientes (quatro alocadas no GA e quatro no GR) por apresentarem alterações no teste ergométrico (TE) máximo, como doença cardíaca isquêmica e/ou arritmias. Devido à onerosidade do $\mathrm{TE}$, somente o realizaram as pacientes que preencheram os critérios de inclusão e que aceitaram participar do estudo por meio da assinaram do TCLE. As etapas de seleção e inclusão constam na figura 1. 


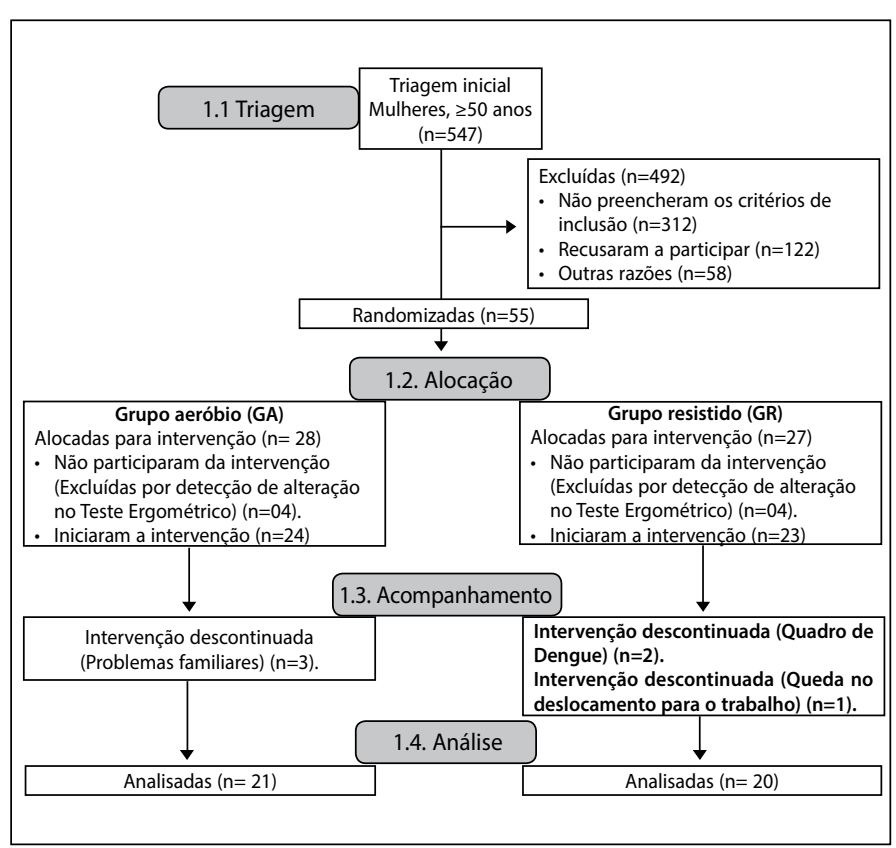

Figura 1. Fluxograma das etapas de seleção e inclusão dos sujeitos participantes do estudo.

\section{Procedimentos de avaliação}

Teste Ergométrico: Realizado em esteira rolante da marca Ecafix, protocolo de Bruce. A interpretação do TE foi realizada com base nas recomendações das II Diretrizes da Sociedade Brasileira de Cardiologia sobre teste ergométrico ${ }^{18}$.

\section{Avaliação sociodemográfica, antropométrica e histórico patológico}

Para a caracterização da amostra, no início (M1), foram investigados, por meio de questionário, dados sociodemográficos: idade, renda familiar per capta (em reais), estado civil (com ou sem companheiro) e escolaridade (em anos de estudo). Foi também avaliada a presença de comorbidades como obesidade e diabetes mellitus. No M1 e após a intervenção (M2) foram coletados: peso com balança eletrônica Filizola ${ }^{\circledR}$, (modelo plataforma, com capacidade para 200 kg e precisão de 100 g, Brasil), estatura (utilizada haste graduada da balança, com precisão de 0,1 cm), circunferência de cintura (CC) com fita métrica inextensível, medida no ponto médio entre a crista ilíaca anterior superior e a última costela ${ }^{19}$ e pressão arterial (PA) com aparelhos semiautomáticos validados da MARCA OMRON - HEM 705 CP. O Índice de massa corporal (IMC) foi obtido dividindo-se o peso em quilogramas pelo quadrado da estatura em metros $^{19}$.

A QV foi avaliada no M1 e M2, por método de entrevista, através do questionário WHOQOL-bref, e a qualidade de vida relacionada à saúde (QVRS) pelo SF-36 ${ }^{16)}$ O WHOQOL-bref, desenvolvido pela OMS, validado no Brasil por Fleck ${ }^{17}$, é composto por 26 questões e abrange os domínios: físico, psicológico, de relações sociais e de meio ambiente. Para sua pontuação é feito um cálculo dos escores para cada domínio, que variam de zero a 100, em que zero corresponde a um pior nível de QV e 100 a um melhor nível. O SF-36 - The Medical Outcomes Study 36 - Item Short Health Survey, avalia a qualidade de vida relacionada à saúde (QVRS), é constituído por 36 itens, englobados em oito domínios: capacidade funcional, aspectos físicos, dor, estado geral de saúde, vitalidade, aspectos sociais, aspectos emocionais e saúde mental. Para a sua avaliação é dado um escore para cada questão que, posteriormente, é transformado numa escala de 0 a 100, em que zero corresponde a um pior nível de QVRS e 100 a um melhor nível ${ }^{16}$.

A capacidade funcional foi avaliada no M1 e M2 pela distância percorrida (em metros) no teste de caminhada de seis minutos (TC6 ${ }^{\prime 20}$.

Realizadas duas sessões de exercícios por semana com duração de 60 minutos cada por um período de oito semanas, perfazendo 18 sessões no total. Os grupos foram acompanhados, no período matutino, em um parque na região central da cidade com pista de caminhada e área apropriada aos exercícios resistidos. A prescrição dos exercícios foi individualizada e os grupos formados em média por seis pacientes. Em cada sessão foi feito controle dos sinais vitais - aferição da PA e da FC, na posição sentada, utilizando aparelhos semiautomáticos da MARCA OMRON - HEM 705 CP, antes de iniciar os exercícios, com cinco minutos de repouso, e cinco minutos após o término dos exercícios. Foi seguida a recomendação ${ }^{5}$ de não iniciar a sessão caso a pressão arterial sistólica e diastólica estivessem superiores a 160 e/ou 105 mmHg respectivamente. Somente foram consideradas para análise as pacientes que apresentaram 80\% de frequência às sessões (mínimo de 14 sessões).

Grupo aeróbio (GA): A intensidade dos exercícios do GA foi estabelecida na faixa de leve a moderada, até $80 \%$ da FC máxima ${ }^{5}$, e monitorada pela escala de Borg adaptada ${ }^{21}$. Realizados alongamentos para os membros superiores e inferiores duas vezes por sessão, após aquecimento inicial de 10 minutos e ao final dos exercícios. Utilizado ainda o controle pela ventilação, ou seja, considerou-se o limite (exaustão) quando cada paciente não conseguia falar frases completas sem interrupções e foi feita orientação para diminuição da intensidade após este limiar ${ }^{5}$.

Grupo resistido (GR): O GR realizou o teste de uma repetição máxima (1RM) para a identificação do nível de força máxima e prescrição da intensidade de cada exercício, estabelecida em até 50\% de 1 RM nas primeiras nove sessões e até $65 \%$ nas nove sessões subsequentes ${ }^{5}$. As sessões foram iniciadas com aquecimento específico (cinco repetições em cada exercício), seguido de alongamentos para membros superiores e inferiores. Em seguida realizadas duas séries com 12 a 15 repetições, com intervalo de descanso de 60 segundos entre as séries e, ao final, alongamentos para todos os grupos musculares. Exercícios realizados: crucifixo com halteres; elevação frontal de ombros com halteres; rosca bíceps com halteres; extensão unilateral de cotovelos com haltere; puxada horizontal com um haltere; extensão de joelho, sentado, com caneleira; flexão de joelho, em pé, com caneleira; abdução de coxa, em pé, com caneleira; adução de coxa, em pé, com caneleira; flexão de tronco; agachamento com pés paralelos ${ }^{22}$.

\section{Análise estatística}

Os dados coletados foram digitados no programa Microsoft Excel e analisados no programa SPSS (versão 16.0, Chicago, IL, USA). O teste Shapiro-Wilk foi usado para verificar se os dados numéricos apresentaram distribuição normal. Para a análise comparativa foram utilizados os testes t-student (dados com distribuição normal) e os testes Mann-Whitney U e Wilcoxon para dados sem distribuição normal. A análise de dados categóricos foi feita com os testes de Fisher e Qui-quadrado de Pearson. $O$ nível de significância adotado foi de $p<0,05$.

\section{RESULTADOS}

Das 47 pacientes incluídas, três alocadas no GA não completaram o treinamento por problemas familiares e três pacientes saíram do GR, pois duas apresentaram quadro de dengue e uma sofreu uma queda no deslocamento para o trabalho. A amostra total analisada foi de 41 mulheres hipertensas. A adesão às sessões no GR foi de 97\% e 96\% no GA, considerando-se o total de 18 sessões.

A tabela 1 mostra que inicialmente os grupos eram homogêneos quanto às características clínicas, antropométricas, funcionais e sociodemográficas ( $p \geq 0,05)$. Pelo WHOQOL-bref, os grupos não eram homogêneos apenas com relação aos domínios psicológico $(p=0,008)$ e pessoal $(p=0,028)$, sendo que o GR tinha escores mais elevados nesses aspectos. Na avaliação pelo SF-36, os grupos eram inicialmente diferentes, apenas quanto aos aspectos físicos $(p=0,018)$, sendo que 0 GA apresentou um maior escore neste domínio. 
Na comparação intragrupo houve melhora significativa em todos os domínios do WHOQOL-bref após o período de intervenção no GA. Não observamos o mesmo no GR, que apresentou melhora apenas no domínio físico (tabela 2).

Pelo SF-36, na avaliação intragrupo, tanto no GA quanto no GR foram encontradas mudanças significativas em todos os domínios, exceto no domínio saúde mental (tabela 3).

Na comparação entre os grupos, no M2 não foram verificadas diferenças significativas nos escores tanto do WHOQOL-bref quanto do SF-36 ( $p \geq 0,05)$.

Com relação às variáveis antropométricas (peso, IMC, CC), clínicas (PA e FC) não foram verificadas mudanças significativas no M2 ( $p \geq 0,05)$ em nenhum dos grupos.

Porém, em relação à capacidade funcional, houve melhora significativa em ambos os grupos, verificado devido ao aumento da distância percorrida no TC6' (figura 2).

Tabela 1. Caracterização inicial da amostra.

\begin{tabular}{|c|c|c|c|}
\hline & $\mathrm{GA}(\mathrm{n}=21)$ & $G R(n=20)$ & p \\
\hline Idade (anos)* & $65,7 \pm 6,9$ & $65,7 \pm 9,9$ & 0,982 \\
\hline Escolaridade (anos)* & $4,9 \pm 3,6$ & $4,1 \pm 3,7$ & 0,491 \\
\hline Renda per capta $(R \$)^{*}$ & $228,0 \pm 189,9$ & $217,9 \pm 139,1$ & 0,847 \\
\hline Com companheiro $^{\ddagger}$ & $12(57,1 \%)$ & $8(40,0 \%)$ & 0,272 \\
\hline Peso $(\mathrm{kg})^{*}$ & $65,7 \pm 15,2$ & $63,9 \pm 7,9$ & 0,636 \\
\hline Estatura $(m)^{*}$ & $1,51 \pm 0,0$ & $1,51 \pm 0,0$ & 0,821 \\
\hline $\mathrm{IMC}\left(\mathrm{kg} / \mathrm{m}^{2}\right)^{*}$ & $28,6 \pm 6,6$ & $27,7 \pm 3,6$ & 0,877 \\
\hline Pressão arterial sistólica $(\mathrm{mmHg})^{*}$ & $130,9 \pm 16,6$ & $127,5 \pm 16,1$ & 0,508 \\
\hline Pressão arterial diastólica $(\mathrm{mmHg})^{*}$ & $78,7 \pm 7,3$ & $75,9 \pm 9,5$ & 0,507 \\
\hline Circunferência da cintura $(\mathrm{cm})^{*}$ & $92,8 \pm 11,3$ & $94,8 \pm 6,9$ & 0,507 \\
\hline Frequência cardíaca (bpm*) & $75,7 \pm 17,0$ & $69,9 \pm 13,6$ & 0,233 \\
\hline Distância percorrida no TC6 $(m)^{*}$ & $395,3 \pm 51,0$ & $386,7 \pm 63,6$ & 0,634 \\
\hline Obesidade $(\%)^{\dagger}$ & $6(33 \%)$ & $5(25 \%)$ & 0,734 \\
\hline Diabetes mellitus $(\%)^{\dagger}$ & $6(33 \%)$ & $4(22 \%)$ & 0,719 \\
\hline
\end{tabular}

*Teste $t$-student para amostras independentes; ${ }^{\top}$ Teste de Fisher; Valores expressos em média \pm desvio-padrão e em percentual. GA-grupo aeróbio; GR-grupo resistido; IMC-I Indice de massa corporal; PAS- Pressão arterial sistólica; PAD- Pressão arterial diastólica; CC - Circunferência da Cintura; FC- Frequência cardíaca; TC6'-Teste de caminhada de seis minutos.

Tabela 2. Avaliação intragrupo dos escores do WHOQOL-bref, grupos aeróbio (GA) e resistido (GR) nos momentos inicial (M1) e final (M2) da intervenção.

\begin{tabular}{c|c|c|c|c|c|c}
\hline Domínios & $\begin{array}{c}\text { GA (M1) } \\
(\mathbf{n = 2 1 )}\end{array}$ & $\begin{array}{c}\text { GA (M2) } \\
(\mathbf{n = 2 1 )}\end{array}$ & $\mathbf{p ~ ( G A )}$ & $\begin{array}{c}\text { GR (M1) } \\
(\mathbf{n = 2 0})\end{array}$ & $\begin{array}{c}\text { GR (M2) } \\
(\mathbf{n = 2 0 )}\end{array}$ & $\mathbf{p}$ (GR) \\
\hline Físico & $55,0 \pm 12,6$ & $65,6 \pm 12,8$ & $0,000^{*}$ & $55,2 \pm 13,4$ & $66,4 \pm 15,9$ & $0,001^{*}$ \\
\hline Psicológico & $56,7 \pm 16,6$ & $64,9 \pm 19,7$ & $0,009^{*}$ & $69,0 \pm 11,1$ & $70,8 \pm 14,0$ & $0,456^{*}$ \\
\hline Relações sociais & $59,5 \pm 15,6$ & $67,1 \pm 23,5$ & $0,027^{\dagger}$ & $71,2 \pm 17,2$ & $70,8 \pm 14,0$ & $0,908^{*}$ \\
\hline Meio ambiente & $50,3 \pm 8,6$ & $60,1 \pm 11,5$ & $0,001^{*}$ & $56,1 \pm 14,6$ & $60,3 \pm 17,1$ & $0,327^{*}$ \\
\hline
\end{tabular}

Tabela 3. Avaliação intragrupo dos escores do SF-36, grupos aeróbio (GA) e resistido (GR) nos momentos inicial (M1) e final (M2) da intervenção.

\begin{tabular}{|c|c|c|c|c|c|c|}
\hline Domínios & $\begin{array}{c}\mathrm{GA}(\mathrm{M} 1) \\
(\mathrm{n}=21)\end{array}$ & $\begin{array}{c}\text { GA (M2) } \\
(n=21)\end{array}$ & $p(G A)$ & $\begin{array}{c}\text { GR (M1) } \\
(n=20)\end{array}$ & $\begin{array}{c}\mathrm{GR}(\mathrm{M} 2) \\
(\mathrm{n}=20)\end{array}$ & $p(G R)$ \\
\hline $\mathrm{CF}$ & $62,8 \pm 18,2$ & $75,7 \pm 15,2$ & $0,006^{*}$ & $59,0 \pm 20,7$ & $71,2 \pm 19,5$ & $0,007^{*}$ \\
\hline$A F$ & $60,7 \pm 42,2$ & $80,9 \pm 24,8$ & $0,033^{*}$ & $28,7 \pm 35,6$ & $68,7 \pm 36,1$ & $0,002^{*}$ \\
\hline D & $55,6 \pm 22,5$ & $68,1 \pm 19,4$ & $0,007^{*}$ & $47,3 \pm 19,5$ & $62,7 \pm 19,6$ & $0,004^{*}$ \\
\hline EGS & $67,2 \pm 19,7$ & $79,6 \pm 14,2$ & $0,013^{*}$ & $63,3 \pm 23,0$ & $74,3 \pm 20,8$ & $0,010^{*}$ \\
\hline V & $55,9 \pm 25,2$ & $71,6 \pm 23,3$ & $0,001^{*}$ & $54,5 \pm 21,5$ & $74,0 \pm 22,5$ & $0,007^{*}$ \\
\hline AS & $60,7 \pm 28,0$ & $89,2 \pm 15,4$ & $0,000^{*}$ & $64,3 \pm 24,0$ & $81,2 \pm 23,8$ & $0,045^{*}$ \\
\hline$A E$ & $52,3 \pm 42,9$ & $76,1 \pm 30,0$ & $0,021^{*}$ & $46,6 \pm 39,5$ & $73,3 \pm 36,8$ & $0,046^{*}$ \\
\hline SM & $60,5 \pm 21,1$ & $69,5 \pm 24,3$ & 0,108 & $63,4 \pm 21,3$ & $71,8 \pm 22,0$ & 0,116 \\
\hline
\end{tabular}

Teste de Wilcoxon; Valores expressos em média \pm desvio-padrão; CF - capacidade funcional; AF - aspectos físicos; D- dor;EGS- estado geral de saúde;V- vitalidade; AS- aspectos sociais; AE- aspectos emocionais; SMsaúde mental; * $p \leq 0,05$.

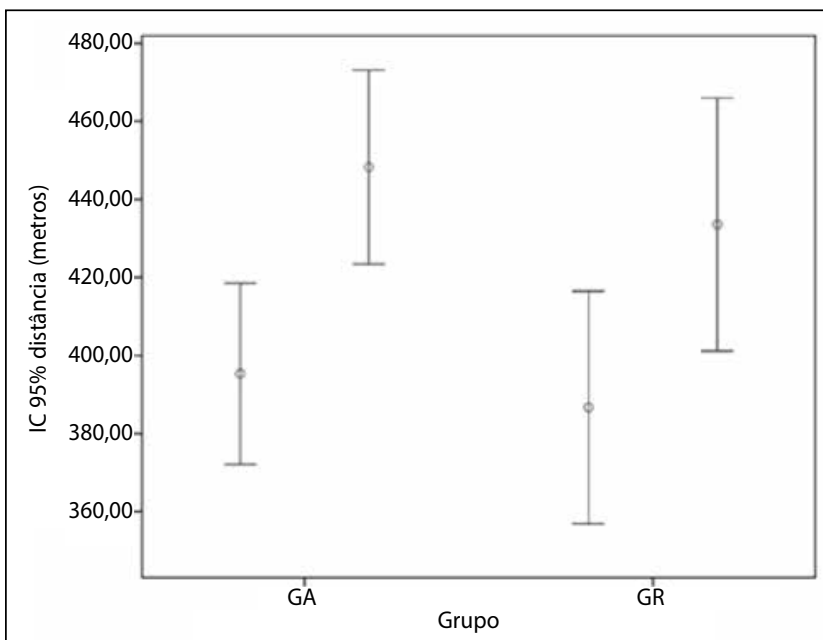

Teste $t$ student para amostras pareadas.

Grupo Aeróbio (GA) - $p<0,001$ e Grupo Resistido (GR) - $p=0,001$

Figura 2. Comparação da distância percorrida pelos dois grupos no teste de caminhada de 6 minutos (TC6 ) em M1(antes da intervenção) e M2 (após intervenção).

\section{DISCUSSÃO}

Os resultados deste estudo indicaram melhora em aspectos importantes da QV (WHOQOL-bref) $)^{17}$ e QVRS (SF-36) ${ }^{16}$ após os protocolos com exercícios aeróbios e resistidos. Foram verificadas algumas diferenças intragrupo pós-intervenção (M2), pois o grupo aeróbio (GA) apresentou melhora em todos os domínios e o grupo resistido (GR), melhora apenas no domínio físico. Contudo, antes da intervenção, o GR já apresentava escores melhores nos domínios pessoal e psicológico em comparação ao GA, o que sugere que pode ter havido uma aproximação dos resultados nos dois grupos na avaliação final (M2). Esta hipótese pode ser pertinente, na medida em que quanto à QVRS, foi observada melhora quase idêntica nos dois grupos no $\mathrm{M} 2$, em que houve aumento expressivo em quase todos os domínios do SF-36, exceto o domínio saúde mental.

Nos dois grupos, houve melhora nos aspectos físicos na avaliação da $\mathrm{QV}$, o que pode ter tido relação com a melhora da capacidade funcional, verificada neste estudo pelo aumento na distância percorrida no TC6 em ambos os grupos. Melhora da QV em paralelo a um aumento da CF também foi detectada por Belardinelli ${ }^{23} \mathrm{em}$ pacientes com insuficiência cardíaca crônica e por Tsai ${ }^{8}$ com hipertensos, os quais verificaram efeitos muito semelhantes aos deste estudo, com melhora em quase todos os domínios do SF-36, exceção para o domínio saúde mental.

Em populações hipertensas, a avaliação da QV tem sido visualizada como ferramenta adicional para a prática clínica e para a escolha de tratamentos com melhor tolerância pelos pacientes, além de ser importante para verificar os efeitos da doença e refletir características não detectadas por medidas clínicas ${ }^{24}$. Na metanálise de Gillison ${ }^{25}$, foram avaliados 56 ensaios clínicos randomizados (ECR`s) e após o treinamento com exercícios no geral, foram verificadas discretas melhoras na QV geral no grupo de pessoas com patologias em relação aos grupos controles. Foram encontradas heterogeneidades quanto aos efeitos dos exercícios sobre os aspectos da qualidade de vida, o que pode ter ocorrido devido às diferenças de objetivos e metodologias dos ECR's analisados, referem os autores.

Há poucos estudos que investigam a influência de variáveis do treinamento importantes como intensidade, frequência semanal e duração sobre a QV. As evidências indicam que altas intensidades provocam efeitos negativos, por desencadearem desconfortos musculo articulares e alterações no humor ${ }^{26}$. Com base nestas constatações e 
na recomendação ${ }^{5}$ de se evitar exercícios vigorosos em hipertensos, optou-se por utilizar as intensidades leve a moderada nos protocolos do presente estudo, o que corrobora com os resultados evidenciados na metanálise de Gillison ${ }^{25}$.

Sobre a influência da frequência e duração semanal sobre a QV verifica-se também escassez de evidências. Há, no entanto, sinalizações de que quanto maior a duração semanal, melhores podem ser os efeitos na QV e na QVRS. Em um ECR, Martin ${ }^{27}$, comparou os efeitos de diferentes durações semanais de exercícios gerais, sobre a QVRS (SF-36), no qual um grupo realizou exercícios durante $72 \mathrm{~min} / \mathrm{semana}$; outro 135 min/semana e o último 190 min/semana. Na comparação com um grupo controle após seis meses, todos os grupos experimentais melhoraram a QV, sendo que aqueles que praticaram exercícios por períodos mais prolongados apresentaram melhores escores no SF-36. Os autores inferiram que a melhoria na QVRS foi dose-dependente do tempo de exercício realizado semanalmente. Contudo, é importante ressaltar que estes benefícios podem não ocorrer caso haja excesso de treinamento ou a presença de limitações osteomusculares e articulares ${ }^{23}$.

Quanto aos domínios psicológico e relacionado ao ambiente da QV, Okuma ${ }^{28}$ refere que os exercícios físicos no geral ampliam as possibilidades de trabalho em grupo, propiciando interação social, conforme realizado nos protocolos de treinamento deste estudo. Segundo estes autores, o grupo possibilita aos seus integrantes adquirir uma identidade social, sentir e ter compromisso com algo e com as pessoas, viver o sentimento de confiança e a relação de companheirismo, importantes para uma melhor QV.

Um ponto importante dos protocolos de exercícios adotados neste estudo foi a viabilidade, já que foram desenvolvidos em local público, com equipamentos e metodologias acessíveis e de baixo custo, pois no GA a intensidade foi controlada pela percepção subjetiva do esforço (PSE), a qual tem tido respaldo ${ }^{21,29}$. Os materiais (pesos livres) do grupo resistido fazem parte do acervo de programas de exercícios de muitas capitais brasileiras, ademais, foram desenvolvidos exercícios com o peso do próprio corpo, como os agachamentos e a flexão de tronco.

Como limitações deste estudo, há o número relativamente reduzido, embora estatisticamente representativo, de sujeitos e o tempo relativamente curto de intervenção. Mesmo assim foram verificadas sinalizações de achados importantes, que podem ser mais explorados em outras populações hipertensas em pesquisas futuras.

\section{CONCLUSÕES}

Os dois tipos de treinamento, aeróbio e resistido, promoveram melhoria em aspectos importantes da qualidade de vida e qualidade de vida relacionada à saúde e na capacidade funcional na população de mulheres hipertensas avaliadas neste estudo.

\section{AGRADECIMENTOS}

À equipe multiprofissional da Liga de Hipertensão Arterial do Hospital das Clínicas da Universidade Federal de Goiás, Goiânia (GO) Brasil, e aos pacientes que participaram deste estudo.

Todos os autores declararam não haver qualquer potencial conflito de interesses referente a este artigo.

\section{REFERÊNCIAS}

1. World Health Organization. The world health report- Life in the 21st century: A vision for all. Geneva, 1998, p.87.

2. Grace SL, Fry R, Cheung A, Stewart DE. Cardiovascular Disease. BMC Womens Health. 2004;4:1-9.

3. American Heart Association. Evidence-based guidelines for cardiovascular disease prevention in Women:2007 Update. Circulation, v.20, p.1-21,2007.

4. American College of Sports Medicine. Position stand: Exercise and Hypertension. Med Sci Sports Exerc. 2004;36:533-53

5. Sociedade Brasileira de Cardiologia, Sociedade Brasileira de Hipertensão, Sociedade Brasileira de nefrologia. VI Diretrizes Brasileiras de Hipertensão Arterial. Rev Bras Hipertens. 2010;17:1-63.

6. Whelton SP, Chin A, Xin X, He J. Effect of aerobic exercise on blood pressure: a meta-analysis of randomized, controlled trials. Ann Intern Med. 2002;136:493-503.

7. Martim JE, Dubbert, PM, Cushman, WC. Controlled trial of aerobic exercise in hypertension. Circulation 1990;81:1560-7

8. Tsai JC, Yang HH, Wang WH, Hsieh MH, Chen PT, Kao CC et al. The beneficial effect of regular endurance exercise training on blood pressure and quality of life in patients with Hypertension. Clin Experiment Hypertens. 2004;26:255-65.

9. Mediano MFF, Paravidino V, Pontes RSFL, Polito MD. Comportamento subagudo da pressão arterial após o treinamento de força em hipertensos controlados. Rev Bras Med Esporte. 2005;11:337-40.

10. Fisher MM. The effect of resistance exercise on recovery blood pressure in normotensive and borderline hipertensive women. J Strength Cond Res. 2001;15:210- 6.

11. Cunha RM, Jardim PCBV. Subacute blood pressure behavior in elderly hypertensive women after resistance exercise session. J Sports Med Phys Fitness. 2012;52:1-2.

12. Polito MD, Farinatti PTV. Comportamento da pressão arterial após exercícios contra-resistência: uma revisão sistemática sobre variáveis determinantes e possíveis mecanismos. Rev Bras Med Esporte. 2006:12:386-92.

13. Spirduso WW. Physical dimensions of aging. Champaign, Human Kinetics, 1995;p. 367-87.

14. Ogunlana MO, Adedokun B, Dairo MD, Odunaiya NA. Profile and predictor of healthrelated quality of life among hypertensive patients in south-western Nigeria. BMC Cardiovasc Disord. Nigéria. 2009:9:1-8.
15. Ayedemir O, Ozdemir S, Koroglu E. The Impact of Co-Morbid Conditions on the SF- 36: A PrimaryCare-Based Study Among Hypertensives. Arch Med Res.2005;36:136-41.

16. Ciconelli RM, Ferraz MB, Santos W, Meinão I, Quaresma MR. Translation into Portuguese and validation of the generic questionnaire for assessing quality of life SF- 36. Rev Bras Reumatol. 1999;39:143-50.

17. Fleck MPA, Lousada S, Xavier M, Chachamovich E, Vieira G, Santos L, et al. Application of the Portuguese version of the abbreviated instrument of quality life WHOQOL-bref. Rev Saude Publica. 2000;34:178-83

18. Sociedade Brasileira de Cardiologia. II Diretrizes da Sociedade Brasileira de Cardiologia sobre teste ergométrico. Arq Bras Cardio.l 2002; 78 (Supl II): 1-18.

19. World Health Organization. Physical status: the use and interpretation of anthropometry. Geneva: WHO,1995; p.10.

20. American Thoracic Society. Statement: Guidelines for the Six-minute walk test. Am J Respir Crit Care Med. 2002;166:111-17.

21. Borg GAV. Psychophysical bases of perceived exertion. Med Sci Sports Exerc. 1982;14:377-81.

22. Fleck S, Kraemer WJ. Designing Resistance Training Programs. 3rd edition.Human Kinetics. 2006;p.326-29.

23. Belardinelli R, Demetrios G, Cianci G, Purcaro A. Randomized, controlled trial of long-term moderate exercise training in chronic heart failure: effects on functional capacity, quality of life, and clinical outcome. Circulation.1999;99:1173-82.

24. Laforest L, Ganse EV, Ritleng C, Desamericq G, Letrilliart L, Moreau A, et al. Correlates of quality of life of pre-obese and obese patients: a pharmacy-based crosssectional survey. BMC Public Health.2009;9:1-11.

25. Gillison FB, Skevington SM, Sato A, Standage M, Evagelidou S.The effects of exercise interventions on quality of life in clinical and healthy populations: a metaanalysis. Soc Sci Med. 2009;68:1700-10.

26. Bowen DJ, Fesinmeyer MD, Yasui Y, Tworoger S, Ulrich CM, Irwin ML, et al. A Randomized trial of exercise in sedentary middle aged women: effects on quality of life. Int J Behav Nutr Phys Act. 2006;3:1-9.

27. Martin CK, Church TS, Thompson AM, Earnest CP, Blair SN. Exercise dose and quality of life: Results of a randomized controlled Trial. Arch Intern Med. 2009;169:269- 78

28. Okuma SS. O idoso e a atividade física. 1 ed. Campinas: Papirus;1998. 208 p.

29. Carton RL, Rhodes EC. A critical review of the literature on ratings scales for perceived exertion. Sports Med. 1985;2:198-222 